\title{
BELL LET'S TALK: A CATALYST FOR SUPPORT AND SELF-DISCLOSURE, OR CORPORATE GREED?
}

\author{
by \\ Harleen Dhami \\ Hons. BA, Communication Studies, McMaster University, 2019 \\ An MRP \\ presented to Ryerson University \\ in partial fulfillment of the \\ requirements for the degree of \\ Master of Professional Communication \\ in the program of \\ Professional Communication
}

Toronto, Ontario, Canada, 2020

(C) Harleen Dhami, 2020 


\begin{abstract}
AUTHOR'S DECLARATION
I hereby declare that I am the sole author of this MRP. This is a true copy of the MRP, including any required final revisions. I authorize Ryerson University to lend this MRP to other institutions or individuals for the purpose of scholarly research. I further authorize Ryerson University to reproduce this MRP by photocopying or by other means, in total or in part, at the request of other institutions or individuals for the purpose of scholarly research. I understand that my MRP may be made electronically available to the public.
\end{abstract}




\begin{abstract}
For years, the stigma around depression has caused many to suffer in silence. Since its launch in 2010, the Bell Let's Talk campaign, started by Canadian telecommunications giant Bell, has aimed to change the narrative around mental health. With Bell coming under fire for overlooking employee mental health needs and even firing staff as a result of requesting time off, this Major Research Paper explores how the 2020 Bell Let's Talk campaign mobilizes support and selfdisclosure among Twitter users or whether it is simply another instance of corporate profitization. Analyzing tweets one week before, the day of Bell Let's Talk and one week after, it is suggested that the campaign does not instill a significant increase in supportive tweets on the day of. Rather, it appears that users engage in self-disclosing their experiences with depression and share resources and ways to cope on the day of the initiative. Comparatively, self-disclosure and support does not appear to be sustained beyond the day of the initiative.
\end{abstract}




\section{ACKNOWLEDGEMENTS}

I would like to extend my heartfelt thanks to Dr. Yukari Seko for the countless hours spent reviewing, editing and helping me form this MRP. Your empathy, support and guidance has been much appreciated. I would also like to thank Dr. Wendy Freeman for serving as a second reader, whose valuable and insightful input helped me create the MRP that stands today.

And to all the mental health workers: thank you. The world is clearly in a state of flux right now — but with your grit, integrity and commitment to helping our communities, you have mitigated some of the uncertainty that many face. Thank you for all that you do. 


\section{TABLE OF CONTENTS}

1. INTRODUCTION 1

2. LITERATURE REVIEW

2.1 Mobilizing open dialogue and peer to peer communication systems on social media 5

2.2 Overview of corporate mental health awareness campaigns on social media 7

2.3 Overview of Bell Let's Talk and 2020 campaign 8

2.4 Pros and cons of Bell Let's Talk

3. RESEARCH QUESTION AND RATIONALE 14

4. HYPOTHESES 16

5. METHODOLOGY 18

5.1 Data collection $\quad 18$

5.2 Method of analysis 19

6. FINDINGS AND DISCUSSION 21

6.1 Hypothesis \#1 22

6.2 Hypothesis \#2 26

6.3 Hypothesis \#3 31

6.4 Hypothesis \#4 33

7. STUDY IMPLICATIONS

8. LIMITATIONS AND FUTURE RESEARCH DIRECTION 36

9. CONCLUSION 38

$\begin{array}{ll}\text { REFERENCES } & 43\end{array}$ 


\section{LIST OF TABLES}

Table 1: type of supportive tweet 23

Table 2: type of self-disclosure in tweets 26 


\section{LIST OF FIGURES}

Figure 1: user segmentation on Bell Let's Talk day 22

Figure 2: volume of supportive tweets per day 23

Figure 3: breakdown of supportive tweets on Bell Let's Talk day 25

Figure 4: self-disclosure versus social awareness tweet breakdown per day 30

Figure 5: example of a tweet with inspirational imagery 32

Figure 6: an example of how corporations use Twitter to advertise their services 33

Figure 7: visual representation of promotional tweets per each day 34 


\section{LIST OF APPENDICES}

Appendix 1: codebook

Appendix 2: type of support

Appendix 3: type of self-disclosure 


\section{INTRODUCTION}

In 2018, 1.6 million Canadians reported that their mental health needs were unmet (Mood Disorders Society of Canada, 2019). Adding to this, $4.7 \%$ of Canadians met the criteria for a major depressive episode (Mood Disorders Society of Canada, 2019).

For far too long, the stigma around depression has silenced those in suffering, whether it be among immediate family, friends, or in the workforce. Rather than seeking out necessary help, many may overlook the severity of their mental wellbeing. In a study by the National Center for Health Statistics (NCHS) at the Centers for Disease Control and Prevention, of the number of depressed Americans over the age of 12, only 35\% saw a mental health professional in the past year. Despite having severe symptoms of depression, the remaining 65\% did not seek professional help (Park, 2014). In 2017, 86\% of Canadians over the age of 12 reported that they had not seen or spoke to a mental health professional about their mental or emotional wellbeing (Statistics Canada, 2017). Given the fact that this study includes the behaviours of pre-teens, this is an especially troubling mindset that many Canadians are adopting from a young age. These figures primarily speak to the fear and stigma associated with asking for help in one's network and within the greater public. On the other hand, accessibility often prevents mental health patients from getting the help they need due to continually expanding waitlists and lack of financial support, to name a few (Social Solutions, n.d.).

Within the last decade, corporations with a large user base have aimed to change how ordinary citizens perceive mental health. Large companies have aligned some of their corporate social responsibility (CSR) initiatives to combat social stigma against depression, with Bell Canada being one of them. Bell was concerned with the extent to which Canadians suffer in silence, due to the stigma and shame associated with mental health. In September 2010, Bell 
Let's Talk was launched as a means of ending the stigma and encouraging informative, respectful conversations among family, friends, co-workers and the greater public. Their mission is to bridge an open discussion around mental illness while offering hope for those who struggle (Bell Canada, "Our Initiatives," n.d.). The premise is simple: citizens are encouraged to text, call or post on social media using the hashtag \#BellLetsTalk. In return, Bell promises to donate five cents to mental health initiatives, such as Kids Help Phone, for every interaction counted. Thus far, the success of Bell's campaign is measured by donations to mental health institutions. They also highlight financial support given to marginalized populations such as Indigenous groups to ensure they have adequate access to services. Bell's total donation to mental health programs since 2010 currently stands at over \$108M (“Our Initiatives," n.d.). Their "Results" webpage states that since 2010 over 790,000 Canadians are supported through the campaign. What is unclear, however, is how exactly individuals are supported (Bell Canada, "Results and Impact," n.d.) and if meaningful change is being made as a result of the campaign.

Bell may be causing more harm than good with its campaign that primarily fuels social media activism, which is then masked as corporate greed. Bell may mean well with their initiative, but more often than not, it mobilizes users to engage in a once-per-year cycle of using the hashtag, shallowly discussing issues online and then moving onto the next trending issue one day after the campaign ends. Bell presents a façade of actively engaging in mental health conversations through their CSR initiative, but this must be more robust and a year-round project for users to see the value in Bell Let's Talk.

Is social media activism, which is essentially what Bell facilitates, just as productive as in-person displays of collectivity? Activism looks different from person to person: some may click a link or tweet with \#BellLetsTalk and others may volunteer at a mental health organization 
or donate to a mental health program. Bell Let's Talk's underlying mechanism that encourages users to engage in supportive, informative ways online is clicktivism. Clicktivism is the intersection of clicking and mediated activism, including using a social button, creating a meme or changing a display icon (Halupka, 2014). Clicktivism is criticized as a feel good and lazy form of activism or a simple mode of participatory culture compared to traditional political participation in public areas. A single click can make users feel as if they have adequately contributed to a campaign's efforts (Halupka, 2014), while in reality, there is often a minimal time commitment involved. Participation in Bell Let's Talk provides little risk to supporters, while the campaign facilitates a mediated form of consumerism. The campaign might be an effortless, easy way for everyday social media users to participate in a worthy cause, while Bell benefits from plenty of free advertising at the same time. So much of Bell's campaign is centered around encouraging open and frequent mental health dialogue among social media users whereas little analysis is provided on whether this is: a) an effective venue for strengthening mediated peer support among social media users; and b) whether users offer advice and support to others via 'likes' and mediated replies.

A similar question can be raised around self-disclosure: to what degree do ordinary users seize this annual opportunity to disclose their experience with depression that would otherwise be seen as taboo? Bell Let's Talk can take advantage of the pre-existing fertile ground for selfdisclosure and support networks that social media facilitate. Social media, and the internet in general, allow for a new form of interaction culture where users can still feel a sense of unity without displaying collective action in person (Gerbaudo, 2012, p. 19). While the campaign may be successful from an advertising point of view, choosing to focus on how users seek relief via Twitter and the extent to which they self-disclose about symptoms, conditions and their personal 
history can determine if Bell's campaign brings real change to the mental health landscape. From here, Bell attempts to mobilize the existing self-disclosure mediated culture to progress with their CSR. These preliminary thoughts and questions have guided this MRP.

This study explores a corporate-sponsored campaign's potential in mobilizing social support and self-disclosure via social media for those who need encouragement during challenging times. 


\section{LITERATURE REVIEW}

In this section I review the extant literature on mental health awareness campaigns on social media and the intersection of peer to peer support, and levels of self-disclosure per theme. The themes that arose include peer-to-peer support on social media, social media as fuel for mental health campaigns and mobilizing support under the façade of CSR. Further, an overview of the 2020 Bell Let's Talk campaign and the initiative's strengths and drawbacks will be reviewed. In what follows, I first describe how peer support is mobilized and strengthened via social media.

\subsection{Mobilizing open dialogue and peer to peer communication systems on social media}

Social media offers a boundless space for users, no matter where they are geographically, to communicate with one another. These bonds can be strengthened as users continue to engage in supportive discourse, especially around sensitive subject matter. Even if you have never met someone in person, social media replicates a similar feeling with an array of ways to engage with user-generated content in affirmative ways.

Research has indicated that social media offers a unique, safe space for users to publicly share their intimate thoughts and challenges with depression. Lachmar et al. (2017) conducted a thematic analysis of 1,978 tweets that contained the hashtag \#MyDepressionLooksLike. Most tweets were categorized under dysfunctional thoughts $(\mathrm{n}=498)$ expressing self-hatred, negativity, how feelings of depression are invalid, and feeling unlovable. Next, the second highest category found was lifestyle challenges $(n=416)$. Here, users would discuss issues with motivation, eating, sleeping and any other daily tasks. The two categories that garnered the fewest tweets were seeking relief $(n=136)$ and suicidal thoughts $(n=110)$. These findings seem to suggest that the 
majority of Twitter users who discuss mental health will not disclose thoughts or personal actions related to self-harm or death, but at the same time, they will not reach out to their network for relief and coping mechanisms. This thematic analysis provides an effective framework for understanding types of self-disclosure that users may engage in, such as dysfunctional thoughts or social struggles. Support and self-disclosure go hand in hand: in order for users to be supportive of one another, users must first self-disclose what they are experiencing in their own lives.

Like Lachmar et al. (2017), Andalibi et al. (2017) employ a visual and textual content analysis of 95,000 Instagram posts to determine: a) the information that people disclose about themselves though depression-tagged posts; b) how captions and images relate to each other; and c) the types of responses (supportive versus unsupportive) that depression-tagged Instagram posts receive. Posts with the hashtag \#depression generally incorporated poor feelings about oneself, stigmatized topics and personal vulnerabilities. Informational support was seen in posts discussing one's self view, where users commented affirmations and supportive discourse. Instrumental support was seen under posts where users disclosed self-harm themes, in which users shared ways to receive help in the comments. Ultimately, Instagram was seen as a place where users could freely disclose stigmatized issues in return for various types of support in the comment section of each post. Andalibi et al.'s (2017) categories of 'support' provide an effective foundation for understanding how users engage with one another on sites that are not temporal in nature, including Twitter. Further avenues to explore in this study may be understanding how the ephemeral nature of Instagram's 'stories' feature affects users' willingness to support one another through resources. 


\subsection{Overview of corporate mental health awareness campaigns on social media}

If social media can facilitate self-disclosure and peer support related to depression, how do corporations such as Bell utilize the platform in their mental health awareness campaigns? Do these campaigns encourage or hinder depression-related self-disclosure and peer support? On the surface, corporate campaigns such as Bell's present an ethically sustainable image that brings good to certain communities. However, there are often underlying motives that companies form their initiative around. Bell is no exception. It was found that CSR initiatives increase customers' perceived value towards a specific brand, while simultaneously boosting trust, commitment and loyalty (Servera-Francés \& Piqueras-Tomás, 2019). These mindsets may then prompt users to continue buying into Bell's services and continue seeing them as the favourable telecommunications provider given their work in the mental health community. These findings also reinforce Jackson's (2018) argument, where users' ability to seamlessly and simply participate from the comfort of their own devices in their homes to then viewing a large company engage in what is perceived as a noble act further entices users to continue engaging with Bell.

The literature provides insight on the effectiveness of corporate campaigns, even over a prolonged period. McClellan et al. (2017) take a longitudinal approach to understand how Twitter users respond to annual events such as Bell Let's Talk versus unanticipated events such as celebrity deaths. Specifically, Bell Let's Talk on January 28th, 2014 was contrasted with Robin Williams' suicide on August 11th, 2014. Looking at 176 million tweets from 2011 to 2014 with suicidal themes, it was found that attention and interest in recurring events fades quickly and is not sustainable since discourse does not last for more than two days. On the other hand, unexpected events lead to more persistent attention and discussion on the part of Twitter users. The study fails to look at events-both expected and unexpected - that are in close proximity to 
one another. It is anticipated that users will not reference Bell Let's Talk day, or the campaign in general, almost seven months later, which the study confirms. Bell Let's Talk may provide users with a range of perceived benefits, including accessible conversations around mental health and a level of anonymity that comes with using social media as a tool for discourse (Chan et al., 2016), however, should unexpected events occur in the campaign period, it is possible that users may gravitate towards these unanticipated moments as opposed to a recurring event.

Perhaps Jackson's (2018) point about ease of access and Chan et al.'s (2016) review of the perceived benefits continue to mobilize users to engage in participatory ways around the campaign. Users' ability to access social media within a limited number of clicks not only creates an unhealthy cycle of 'digital housework' (Peters, 2018) without compensation, but the compounded effects of this ongoing cycle may provide the illusion that users are adequately contributing to a campaign's mission without having to leave their homes. Additionally, the intent of mediated mental health campaigns — to reduce the associated stigma — was likely taken advantage of by Bell in order to raise greater awareness around the campaign. The nature of trending topics on Twitter for example, was one such way that awareness was spread on the day of the virtual event.

\subsection{Overview of Bell Let's Talk and 2020 campaign}

Since its inception in 2010, Bell Let's Talk has always been founded on four central pillars; anti-stigma, care and access, research and workplace health. The campaign's mission is to engage Canadians to take action to form positive change in mental health conversations (Bell Canada, “Our Initiatives,” n.d.). The campaign started as a five-year \$50 million project to help combat the stigma around mental health. In 2015, the campaign was renewed for another five years with a commitment of $\$ 100$ million towards mental health initiatives. Around the time of 
this year's (2020) campaign on January 29th, Bell announced that the initiative was renewed for another five years and would commit \$155 million (“Our Initiatives,” n.d.). This year, the campaign's theme was 'Mental Health: Every Action Counts.'

From 2011 to 2020, the number of interactions that Bell has recorded is currently 1.17 billion (Bell Canada, "Results and Impact," n.d.), which takes into account the number of times users have texted, tweeted or posted using the hashtag \#BellLetsTalk. A noticeable spike in interactions occurred from 2012 to 2013 , where the number of interactions went from 78 million in 2012 to 96 million in 2013 (an increase of 18 million interactions). In recent years, 145 million interactions were recorded in 2019, whereas in 2020, 154 million interactions were counted — an increase of 9 million tweets (Bell Canada, "Results and Impact," n.d.). Though small, the noticeable decline in tweets year-to-year may suggest that users do not solely associate mental health discourse with the campaign anymore as they may once have at the start of the decade.

\subsection{Pros and cons of Bell Let's Talk}

A ten-year campaign like Bell Let's Talk has provided a significant venue for mental health conversations to take place, when they would have otherwise gone unheard. However, does Bell facilitate meaningful, sustainable conversations or is it primarily ephemeral and limited to the two-week campaign period in mid-January?

The material aspect of what Bell Let's Talk has accomplished in the last decade has not gone unnoticed. By mobilizing social media users, Bell has committed over \$108 million in funding toward mental health initiatives (Bell Canada, "Results and Impact," n.d.). By taking advantage of a fertile space for political action and employing hashtag activism, Bell encourages millions each year to continue engaging in sensitive conversations around mental health. The 
nature of social media spreads these conversations into each corner of the digital realm, further promoting mental health-related topics. For instance, the 'trending topics' section of Twitter amplifies the campaign even further, ensuring everyone-even those who were previously unfamiliar with Bell Let's Talk — know what the campaign intends to discuss. Further, seeing a large company like Bell back a worthy philanthropic cause may encourage more informative, socially aware conversations in the workplace and at home. The resources that Bell shares, such as their Conversation Guide on how to facilitate informative discussions with community members, provide tactical tips for strengthening daily encounters with mental wellbeing in mind (Bell Canada, "Ways to Help," n.d.).

The nature of CSR campaigns in general have helped position Bell Let's Talk as a widely discussed and overall successful campaign in terms of user engagement. Like Jackson (2018) notes, CSR campaigns may mobilize users to take part in forms of mediated activism-which have worked to Bell's advantage. Users are first informed about the campaign through advertisements and the trending hashtag on social platforms, thus mobilizing them to share a post with the same hashtag or view a video, where all views count towards donations. Bell has made strategic use of their CSR campaign to: a) increase brand awareness among social media users; and b) boost users' trust and loyalty towards their brand (Servera-Francés \& Piqueras-Tomás, 2019). The strategic use of a CSR campaign powered through social media along with raising awareness through hashtag activism are some of Bell's strongest points associated with Bell Let's Talk.

While Bell touts its campaign as one that brings real change to those suffering from mental illnesses, many have been skeptical of the campaign's effects on ordinary social media users. Peters (2018) notes how racialized and intersectional identities (such as women of colour) 
are often absent from Bell's campaign ads, which generally revolve around the white middle class. From a user standpoint, Bell Let's Talk may cause users to engage in unpaid labour, as is the case with social media campaigns. Affective labour makes for a new generation of exploitive labour whereby users work without recognition or pay and are expected to engage with publics in empathetic ways (Peters, 2018, p. 403).

Clicktivism, as discussed earlier, expends little commitment or time (Halupka, 2014). In the case of Bell Let's Talk, given the five cents donation on Bell's part, users may retweet others instead of sharing their own thoughts in a new tweet. Although users may assume that they have adequately contributed to a CSR initiative through a single click online, how does this make users feel connected to the campaign? Is participating in social media activism enough to make users more informed and comfortable with discussing mental health issues?

Perhaps clicktivism is a low-cost, simple way to encourage ordinary social media users to share information about themselves online while feeling as if they are now part of a nationwide initiative. Disclosing information about oneself online, and on social media in particular, provide a slew of perceived benefits for university-aged students. In focus groups of university students, Chan et al. (2016) discovered these perceived benefits to primarily be anonymity and accessibility when seeking help and voicing concerns at any time of the day. It is possible that users who grapple with depression may be more likely to gravitate towards getting free, informal help on social media regardless of the time of day, as opposed to seeking out professional help in their communities. However, users are still engaging in unpaid, affective labour through their social media interactions. Users engage in exploitative labour and continue to talk about their trajectories online simply because it affords them a level of privacy and anonymity. However, the perceived benefits anticipatedly outweigh the labour component of the equation: being able 
to seek support in an anonymous manner online is enough to draw users to social media versus clinical help elsewhere.

Another criticism toward Bell Let's Talk stems from unjust employee treatment. For this specific group, Bell does more harm than good. In January 2017, former Bell employee Maria McLean (2017) requested time off to deal with her depression. After gaining the confidence to disclose her personal history with her manager, she was fired for unknown reasons and did not receive an explanation as to why she was let go. On a personal level, McLean's prescribed twoweek rest window was bumped to eight due to the trauma and discomfort she experienced with work. McLean is not the only employee that faced discriminatory treatment from Bell. Others like Karen Ho (2016) revealed that as a permalancer - a long-term freelancer - she was not entitled to Bell's Employee Assistance Plan. When Ho realized the toll that a fast-paced job in media took on her body, she managed to get access to the plan through her executive producer. Reflecting on her experience, she specifically identified women of colour as those who are subjected to Bell's unjust treatment, where low pay and no benefits is the acceptable and required corporate ladder to climb in order to land a prestigious job in media.

McLean and Ho's testimonies show how the perceived benefits that Chan et al. (2016) outline also apply to Bell's own employees. On the surface, Bell's initiative may promote accessible discourse around mental health conversations that would have otherwise been seen as taboo. Bell fosters a culture of perceived openness and support, yet when employees take advantage of this, they receive a completely different response. McLean and Ho are the embodiment of prejudicial employee treatment due to taboo, heavily stigmatized topics. Both testimonies suggest that Bell presents an image of solidarity and invites open discourse, but their blatant bias towards their own employees who grapple with mental health issues is quite apparent 
below the surface. Unjust employee treatment may also suggest that Bell's campaign is not as holistic and sincere as it is made out to be.

Others argue that Bell's corporate social responsibility (CSR) façade masks their key revenue-generating measures, especially smartphones. CSR is a business' commitment to invest and nurture the socio-economic effects of its operations sensibly and meet the public's expectations. By engaging in CSR initiatives, companies present an ethical image of themselves to the public, establish good reputations and minimize environmental impacts in the process. Bell hopped onto the CSR bandwagon in 2010 with the launch of Bell Let's Talk. The campaign's perceived success boils down to consumers' ease of participating, Bell's long-term commitment and celebrity endorsements (Jackson, 2018). However, instead of simply being called 'Let's Talk,' 'Bell' was prefixed to the campaign name so users would always be reminded that this was an effort pioneered by the telecommunications company.

Paradoxically, Bell sells smartphones and other electronic products that can contribute to millennials' anxiety. Razak and Aziz (2018) examine perceptions of three social media campaigns, The Power of Okay, Instagram's \#HereForYou campaign and finally, \#IAmNotAshamed, by surveying 100 youth in Malaysia. Around 68\% mention that they have diagnosed themselves with depression since they have never sought professional help. Over half of the respondents also mentioned that the commentary under their post determines their mood and another half also believe that the root cause of depression is, ironically, social media use. These devices can then be leveraged to access the internet and social media, as Chan et al. (2016) note, providing users with the perceived benefit of receiving help within their networks without having to pay. 


\section{RESEARCH QUESTION AND RATIONALE}

After reviewing and critiquing the available literature on the intersection of mental health and social media use, a research question was formed. The research question that will guide this study is: how does the 2020 Bell Let's Talk campaign mobilize self-disclosure and social support among Twitter users?

In this context, support is defined as a gesture of community building through sharing experiences and emotions with others. A supportive tweet may include affirmative, inspirational content and resources on how to cope with depression. Self-disclosure refers to expressing personal sentiment and details one's personal trajectory and experience with depression, if applicable. The following are rationales for why the specific social media platform, campaign and criteria were chosen.

Twitter was chosen due to its lower active user rate compared to other platforms that Bell Let's Talk advertises on. For instance, Twitter has around 300 million active users whereas Instagram has well over 1 billion and Facebook has 2.5 billion (Robinson, 2020). The lower active user rate on Twitter can eliminate unrelated topics under the hashtag. This is one of the only platforms that clearly states trending topics, making it easier to spot campaign-related discourse. It is also anticipated that ephemeral platforms, or the 'stories' feature that many platforms like Facebook and Instagram offer, would not be effective for analyzing sensitive subject matter.

The present study reviews a single campaign in order to ensure all results were as pronounced and nuanced as possible. A drawback to Razak and Aziz's (2018) work, for instance, was non-generalizable results which was a result of analyzing three separate campaigns. A general hashtag like \#depression was also chosen since it invites both critical and optimistic 
discourse and since so many members of Generation $\mathrm{Z}$ do not seek help due to stigma and access barriers. Sample tweets were largely skeptical and critical and did not align with social support themes. Support and self-disclosure were selected in order to build upon the existing literature reviewed. Andalibi et al.'s (2017) categories of support will form the framework for analyzing the types of support that users engage in. Their study was one of the only works to effectively dissect the types of support that users display among their social media networks. On the other hand, Lachmar et al. (2017) offer an effective foundation for understanding how users selfdisclose on Twitter. Their core categories including 'dysfunctional thoughts' and 'lifestyle challenges' will be included, however, other categories such as 'positive self-reflection' and 'social awareness or anti-stigma' will be added to ensure a wide array of themes are adequately represented.

A sub-goal of this study is to examine the correlation, if any, between supportiveness and self-disclosure. For instance, can self-disclosure be supportive or is it largely revealing one's own life in a negative way? By collecting data at three different time points, this study seeks to understand how discourse around depression changes over time with a CSR initiative backed by a large player in the telecommunications industry looming overhead. 


\section{HYPOTHESES}

This section will outline four study hypotheses. These hypotheses were guided by Andalibi et al.'s (2017) work around supportive discourse on depression and Lachmar et al.'s (2017) findings on self-disclosure rates on Twitter. In Chan et al.'s (2016) work, it was made clear that users appreciate and take advantage of the perceived benefits that social media activism affords, including accessible conversations and anonymity depending on one's privacy settings. It is then anticipated that there will be breadth and depth to the tweets gathered at all three time points. Peters' (2018) review of affective labour also reinforces how most social media users will continue to use platforms to engage in meaningful conversations without compensation. The following hypotheses were built off the above literature and context around the intersection of mental health, social media platforms and certain affordances that users are provided.

H1: Bell Let's Talk day will: a) reveal the highest number of supportive tweets; and b) most of which will be categorized under esteem support.

Per Andalibi et al. (2017), esteem support refers to communicating respect and confidence in one's abilities. With targeted advertisements on all social networking platforms, especially more rampant on the day of Bell Let's Talk, it is anticipated that users will engage in supportive communication around mental health issues. Tweets such as 'remember you are not alone' may be grouped in this category. Once Bell Let's Talk day passes, however, with the lack of advertisements and trending topics around the campaign on Twitter, it is anticipated that the number of supportive tweets will fade.

H2: Bell Let's Talk day will: a) see the highest number of self-disclosure tweets; and b) most of which will be categorized under seeking relief. 
Drawing on the work of Lachmar et al. (2017), seeking relief refers to coping mechanisms and addresses ways to alleviate depression. It is anticipated that Bell Let's Talk day will allow users to disclose their personal histories and trajectories around depression. Pertaining to the seeking relief theme, it is expected that users who currently struggle with depression will seek coping mechanisms from others who are in a similar position or in a position to help.

H3: Bell Let's Talk day will see the highest volume of inspirational imagery attached to tweets.

Tying into $\mathrm{H} 1$ where most tweets are anticipated to fall under esteem support, it is expected that this particular day will yield the most supportive imagery attached to tweets. A large part of what Twitter affords includes mixing text and imagery and it is expected that users will take advantage of this on a day when they are encouraged to engage in mediated interactions.

H4: Bell Let's Talk day will see the highest number of advertisements.

Due to the anticipated increase in tweet volume on the day of Bell Let's Talk, it is expected that corporations and services will take advantage of this to promote themselves. Social media has always provided a fertile ground for advertising, but this is even more prevalent on the day of a large campaign that thrives on social media use. 


\section{METHODOLOGY}

\subsection{Data collection}

The campaign's main call to action is to simply talk — whether that be through a phone call, text, tweet or other social media post — to end the stigma around mental illness. A longitudinal approach—spread across three weeks—will allow time for any unexpected events around the campaign period to occur. This may influence the volume of tweets recorded on particular dates that I examine.

Bell also aims to mobilize users to take action to create meaningful change in mental health ("Our Initiatives," n.d.). Considering this, it was anticipated that users would disclose personal experiences to further humanize the narrative around depression. In a similar vein, it was expected that users would demonstrate supportive responses to those struggling with depression where they would validate one another's experiences and share appropriate resources on how to get help.

Although only publicly accessible tweets containing the hashtag \#depression were included in this study, preserving users' privacy is of utmost importance. As such, all personal identifiers including name, API and Twitter username have been removed from the MRP. All tweets were collected between 1 to 2 PM EST on their respective days, accounting for the fact that each day was a weekday and so this period was an anticipated lunch hour for those in EST. All data including copy included in each tweet and publish time are captured in an Excel spreadsheet. Tweets that were not in English were manually translated using Google Translate.

Due to the increase in tweet volume on January 29th, Netlytic - a text and social network analysis tool—was employed to ensure all tweets were captured. I was able to record all publicly available tweets that used the hashtag \#depression along with tweet publish dates, times, 
descriptions and authors - which were later removed. A limitation of Netlytic is tweets are returned from as far as one week prior to the search date. In addition, only the 10 most recent tweets are provided per user. Thus, if a user tweeted 12 times within the hour window, only the first 10 will be documented. Another tool for text grabbing, Snagit, was used pre and post-Bell Let's Talk. I was able to parse all screenshots through Snagit, which generated plain text that was included in each tweet.

\subsection{Method of analysis}

This study employs the method of content analysis to examine tweets with the hashtag \#depression collected at the three specified time points. A codebook developed in collaboration with Dr. Seko guides the content analysis (Appendix 1). The codebook focuses on who tweeted - corporations, individual users or healthcare providers, to name a few. I then examined: 1) support around seeking relief (from depression); and 2) self-disclosure around sensitive issues that users engage in, if at all, through the Tweet Types category. This section along with the first were included to understand whether supportive and unrestricted discourse occurs between users, or from corporations from an advertising standpoint. Co-occurrence of other tags was also examined to provide additional insight into how users viewed their mental well-being: for instance, a popular tag, \#SickNotWeak, may critique the stigma against depression. Finally, examining external sources can demonstrate the extent to which users support one another through websites, online resources or affirmative imagery or statements.

The codebook drew on categories developed by Andalibi et al. (2017) to identify types of 'support' mobilized by \#depression. Categories including 'informational support,' 'instrumental support,' and 'emotional support' were coded (Appendix 2). An additional category for 'social awareness or anti-stigma' tweets was added since not all tweets were for the purpose of support 
as much as awareness. For the second part of my research question which seeks to understand self-disclosure types on Twitter, the codebook built on the various types of self-disclosure outlined by Lachmar et al. (2017) including 'dysfunctional thoughts,' 'lifestyle challenges' and 'seeking relief' (p. 4) (Appendix 3). Based on my preliminary analysis, I added categories including 'mention of specific conditions' and 'positive self-disclosure,' to account for the fact that self-disclosure can be either negative or positive, and not just the former. The category 'social awareness or anti-stigma' as seen in the types of support section was also added here. Analyzing whether or not users engage in conversations around specific conditions can also reveal whether Bell Let's Talk plays a role in encouraging discourse around normally stigmatized topics.

During the process of data analysis, I assessed inter-coder reliability to ensure analytic rigor for both parts of the study. A second coder (Dr. Seko) double-coded a randomly selected $10 \%$ of all the tweets using the same codebook. After an initial round of coding with $55 \%$ alignment on tweets from Bell Let's Talk day, the codebook was refined by discussion until a consensus was reached. Using the modified codebook, our coding achieved the matching rate of $81 \%$ the week before, $70 \%$ on the day of and $78 \%$ one week after. 


\section{FINDINGS AND DISCUSSION}

The data set for this study includes tweets with the hashtag \#depression. Tweets were captured at three separate time points: one week before 2020 Bell Let's Talk $(01 / 22 / 2020)$, the day of $(01 / 29 / 2020)$, and one week after (02/05/2020). Screenshots were taken to preserve both textual and visual semantics of each tweet. 414 tweets were gathered in total, with 66 stemming from the week before, 261 on Bell Let's Talk day and 87 from one week after. Only the initial tweet and first retweet were included, whereas all subsequent retweets $(n=24)$ were removed. The final data set consists of 237 tweets generated on Bell Let's Talk day and a total of 390 tweets were examined. Of the four hypotheses outlined in section four, two were supported and two were not. This section outlines the results from the 390 tweets that were coded in Microsoft Excel and attempts to apply these findings to the broader world of social media users.

First, a demographic review will help contextualize the type of users who tweeted. On the day of Bell Let's Talk, 72\% ( $\mathrm{n}=171)$ of users were identified as individual users with no relationship to any service or brand. On the same day, it appeared that only $6 \%(\mathrm{n}=15)$ of corporations tweeted using the hashtag \#depression. The audience analysis in relation to the findings on the type and quantity of inspirational imagery perhaps suggests that individual users are more likely than any other group to make use of Twitter's platform affordances to share messages of hope and support. Corporations may have been more likely to link to their website to invite viewers to learn more about their product, but individual users were generally the group who participated in the Bell Let's Talk campaign. An overview of the users that tweeted on Bell Let's Talk day is provided in figure 1. 


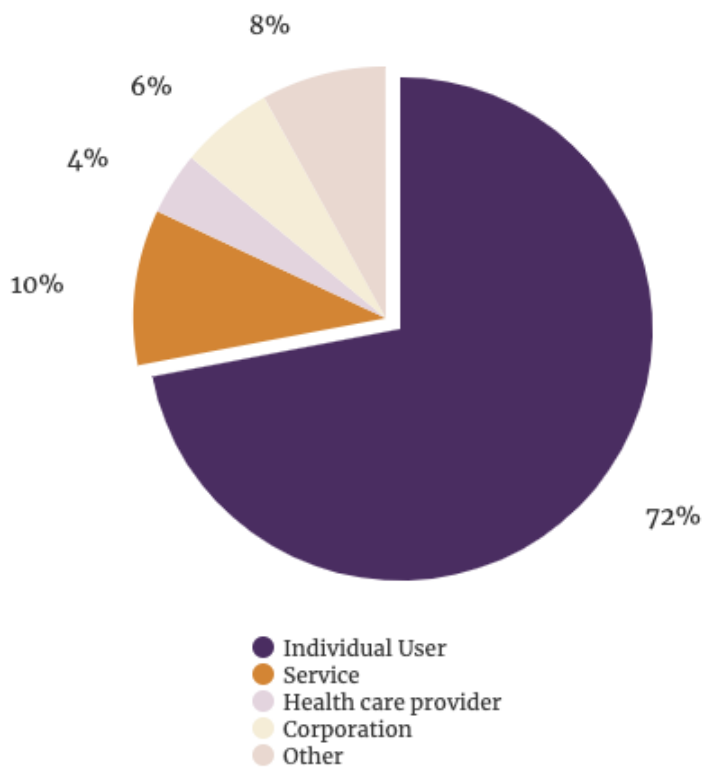

Figure 1: user segmentation on Bell Let's Talk day (1-2 PM EST)

\subsection{Hypothesis \#1}

Hypothesis \#1 (H1) stated that 'Bell Let's Talk day will: a) reveal the highest number of supportive tweets; and b) most of which will be categorized under esteem support.' Esteem support communicates respect and confidence in one's abilities. Both parts of this hypothesis were not supported by the findings. Tweets from the day of Bell Let's Talk, January 29th, were marginally more supportive than one week before the campaign. Supportive tweets made up $11 \%$ $(\mathrm{n}=7)$ of all tweets on January 22nd, and on January 29th, supportive tweets made up 11\% $(n=26)$ of the sample. The percent of supportive tweets increased one week after Bell Let's Talk to $16 \%(\mathrm{n}=14)$ (figure 2$)$. 

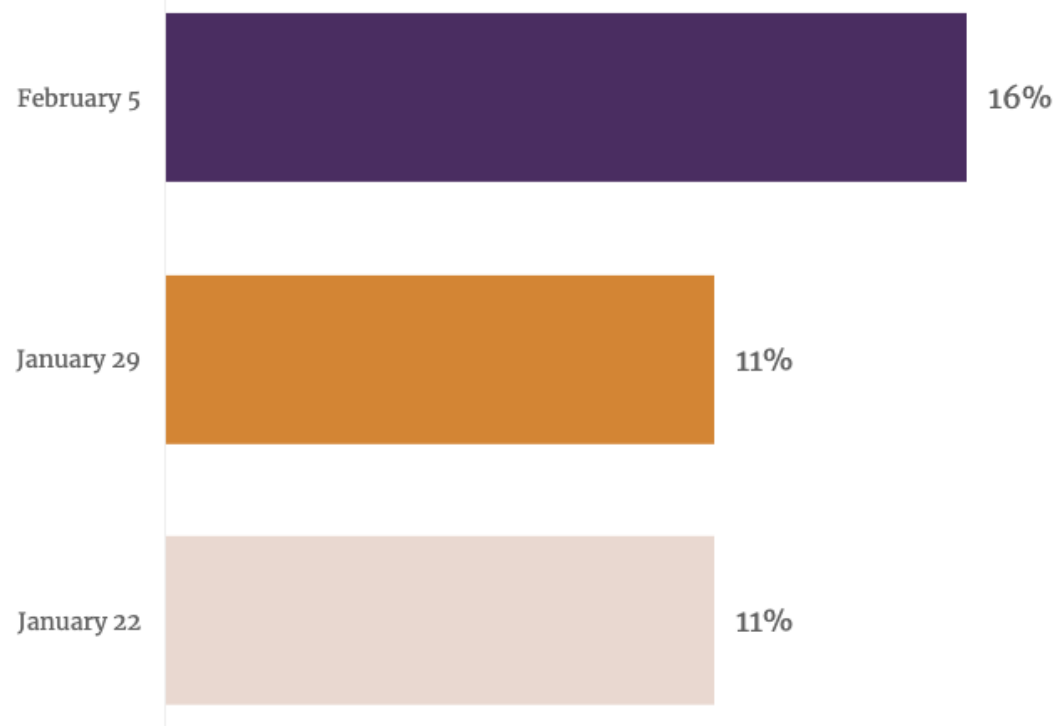

Figure 2: supportive tweets per day

The second part of $\mathrm{H} 1$ looks at the specific types of support that users engage in. The categories of support largely build off of Andalibi et al.'s (2017) categories. A full breakdown of sample tweets for each category are found in table 1. Definitions of each category are provided in the codebook (Appendix 2).

Table 1: Type of Supportive Tweet

\section{Type of Support Example Tweet}

Informational Happy \#WellnessWednesday - here's a great way to stay well this week! support With \#depression and other \#mentalhealth obstacles, it's easy to feel isolated and alone. We encourage you to phone a friend or loved one this week, even if it's just to catch up. tmsmind.com

Instrumental Money cannot buy \#happiness ...

support

Money doesn't guarantee \#Mentalhealth ....

Share your fears \& emotions with someone whom you trust. Fight \#depression and take help of a \#psychologist... be a fighter.

Emotional support I let my mental health tear me and my best friend apart. And since my \#depression has been treated, I make the effort everyday to let him know 
I'll always be his brother, no matter what happens. \#BellLetsTalk

Network support There's nothing wrong with me, and there's nothing wrong with YOU. Being comfortable being me makes me the happiest, conversely, pretending to be someone I'm not drove me to depression. \#Depression isn't a "disease" I contracted, but a reaction to suppressing who I really am.

Esteem support Did you see that? I lost weight! Did you see that? I glow up! Did you see that? I don't need you anymore! \#depression \#depressionquotes \#depressionthoughts \#lost \#fucklove

Unsupportive Show your Valentine you where thinking about them with the gift of relief! \#CBD \#CBDredlands \#redlands \#inlandempire \#cbdoil \#cbdproducts \#1cbdintheie \#deltapremiumCBD \#cbdedibles \#PTSD \#Anxiety \#Depression \#PainRelief \#ValentinesDay

Social awareness RT @ life_iz_short: At this very moment... 3,000,000 \#Canadians are or anti-stigma suffering from \#Depression \#BellLetsTalk \#BellLetsTalkDay \#TikTok...

It was expected that esteem support themes would dominate on Bell Let's Talk day since users would anticipatedly validate one another's experiences while expressing hope and empathy. Surprisingly, the level of esteem support stayed constant across all three days, sitting at $3 \%$ of the number of supportive tweets. The biggest spike in supportive tweets were for informational support on the day of Bell Let's Talk. Informational support in this context provides guidance and advice around a particular facet of mental health struggles. Five percent $(n=3)$ of tweets were categorized under informational support one week before, which jumped to $11 \%(\mathrm{n}=26)$ on the day of the event. It is worth noting that this type of support is not sustainable: one week later, the amount of guidance and advisory themes in tweets drops back to 5\% ( $\mathrm{n}=4)$.

The majority of tweets ( $19 \%$ where $\mathrm{n}=45)$ on Bell Let's Talk day addressed ways to cope and get help, which were categorized as instrumental support. A numeric overview of supportive tweets shared on Bell Let's Talk day is provided in figure 3. Overall, the volume of tweets that address coping mechanisms stays the same across all three dates. However, the volume of 
instrumental support tweets makes up the biggest portion of supportive tweets that were seen on Bell Let's Talk day. In relation to other types of support then, it is clear that users take this day to reach out for help among their networks.

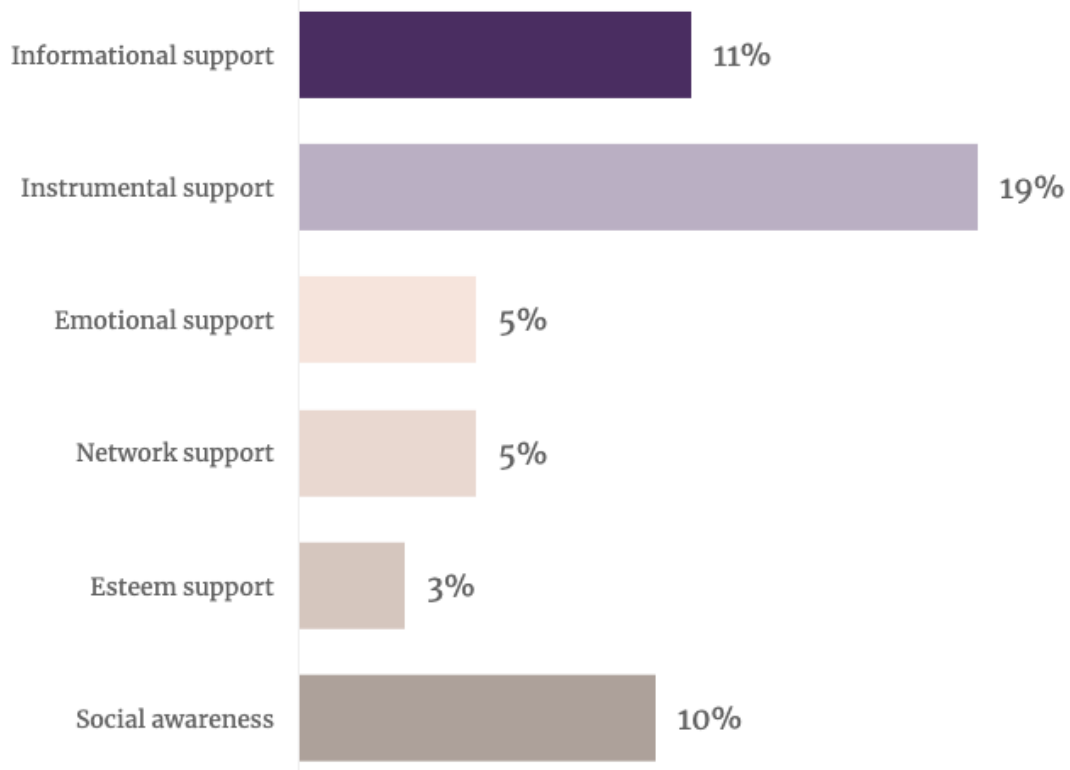

Figure 3: breakdown of supportive tweets on Bell Let's Talk day (January 29, 2020)

All findings were not supportive of H1. It appears that the volume of supportive tweets and the extent to which users communicate confidence in others' abilities is not correlated to a mental health campaign like Bell Let's Talk. The volume of tweets that aligned with esteem support stayed the same across all three dates. Thus, this data suggests that users are not more likely to share affirmative statements via Twitter simply because a corporate campaign tells them to. Equally intriguing is the fact that users share more than two times the amount of informational support in their tweets on the day of the campaign. Five (5) percent $(n=3)$ of tweets included informational support themes one week before, contrasted with $11 \%(n=26)$ on the day of the initiative. This suggests that with all the advertising and broadcasting around supporting peers, users take this as a call to action to directly share their advice and guidance with others. This is only ephemeral in nature, however, since informational support fades just one week after, 
where tweet volume plummets back to $5 \%(\mathrm{n}=4)$. It can also be argued that the awareness component of any CSR campaign is enough to mobilize users to self-disclose and share supportive tweets with the hashtag. It is equally possible that some users first heard of Bell Let's Talk on the day of the virtual event due to the widespread reach that social media affords.

\subsection{Hypothesis \#2}

Hypothesis \#2 (H2) stated that 'Bell Let's Talk day will: a) see the highest number of self-disclosure tweets; and b) most of which will be categorized under seeking relief.' The following analysis was able to support both components of $\mathrm{H} 2$. In this context, self-disclosure refers to elaborating on one's personal trajectory and experience with depression, if applicable. Categories of self-disclosure were adapted from Lachmar et al.'s (2017) study and further built upon for the purpose of this study. Table 2 dissects each category of self-disclosure examined. Detailed definitions of each category can be found in the codebook (Appendix 3).

Table 2: Type of Self-Disclosure in Tweets

\section{Type of Self- $\quad$ Example Tweet \\ Disclosure}

Dysfunctional The bags under my eyes are huge. I look awful right now \#depression thoughts \#insomnia

Lifestyle challenges Filming my vlog then going back to sleep. Been so depressed lately all I wanna do is sleep. \#sleep \#tired \#vlog \#video \#filming \#film \#depression \#mentalhealth \#mentalillness \#mentalwellness

Social struggles Ending The \#Stigma: One in five adults experiences mental illness each year - but we don't often hear people speak openly about their own struggles. https://t.co/WET1VYtcQN \#PSTD, \#mentalhealth , \#Anxiety \#depression \#depressionforums.org

Hiding behind a mask

Emotionally: I'm done. Mentally: I'm drained. Spiritually: I feel dead. Physically: I smile. 


\begin{abstract}
Apathy and sadness "Mind Builder"
I am a proud craftsman

Today

But a shameful prisoner

Of my own mind

Tomorrow.

\#poetry \#poetrycommunity \#amwriting \#mindbuilder \#prisoner \#today \#TOMORROW \#depression
\end{abstract}

Suicidal thoughts and behaviours

User seeks relief

Positive selfreflection

Personal ailments or disorders

No self-disclosure

Social awareness or anti-stigma
Sometimes I feel death is the only solution of all the suffering, pain and expectations.\#depression

Replying to @ StampStigma

Before I was comfortable talking about \#depression, \#anxiety and \#trauma, I found \#poetry to be a great way to express things without doing so obviously. Imagery and word choice painted pictures I couldn't identify or share \#BraveChat

Today I agreed with my therapist that every single day I will list 3 \#positive things. Today I'm grateful for my amazing \#sister, \#fabfriends and my new \#nephew Some days the only positive might be getting my shoes on the right feet but right now I'm feeling it \#depression

Getting thru the morning has end up greater intricate on the grounds that I was identified with hidradenitis suppurativa seven years ago. I typically wake up to discover pus or blood on my pillow and/or sheets. \#anxiety \#ChronicPain \#depression \#dermatol https://t.co/6Qc2kcH7dR https://t.co/c07fQ0KRp5

"By focusing on such a narrow population, Legare and other critics argue, psychology researchers have - mostly unwittingly — presented a skewed view of the human mind" \#psychology \#mentalhealth \#Mindfulness \#anxiety \#depression \#compassion https://t.co/U0qgAsWUiQ

A @ uofg \#mentalhealth survey of Cdn \#farmers: 58\% met the definition of \#anxiety, 35\% met definition of \#depression, $45 \%$ highly stressed, 38\% high levels of emotional exhaustion, and $40 \%$ afraid to seek help because of negative stereotypes! \#ontag \#cdnag this is not ok!!! 
Overall self-disclosure rates were the highest on Bell Let's Talk day $(22 \%$ where $n=51)$. Self-disclosure rates were $15 \%(n=10)$ one week before and $18 \%(n=16)$ one week after. Compared to the findings around supportiveness, this perhaps suggests that while users may be less likely to share affirmations online, they take the opportunity to disclose information about themselves that may otherwise be seen as taboo. Sharing messages about sensitive or heavily stigmatized topics is ultimately one of the pillars of Bell Let's Talk (anti-stigma) and so this disclosure is somewhat anticipated. There is not a significant difference in self-disclosure tweets prior to and post-Bell Let's Talk. Beyond the campaign, it can be inferred that Twitter has already provided a fertile ground for mental health disclosure. Users' voluntary disclosure seems to have had little to do with a well-known CSR initiative. Instead, it is possible that users shared personal information about their mental health histories and journeys since they received resources and guidance from those in their networks, as hypothesis \#1 alluded to.

Next, part b) of this hypothesis that discusses users seeking help was also supported by the findings. According to Lachmar et al. (2017), tweets that align with 'seeking relief' themes refer to self-care and coping mechanisms. Tweets may also include ways to alleviate depression. There is a significant spike in seeking relief themes on the day of Bell Let's Talk $(13 \%$ where $\mathrm{n}=30$ ) compared to a week before $(5 \%$ where $n=3)$. The volume of tweets that reference relief and coping themes significantly drops one week after, where these tweets make up $3 \%(n=3)$. While causation cannot be determined in a study of this size and method, it may be possible that the campaign mobilizes discourse around seeking help, possibly with all the advertisements on Bell's part that push for users to talk about their mental wellbeing. Users may seize this opportunity to go out of their way to provide resources on how to manage their depression. 
For both parts of this hypothesis, anonymity and accessibility (Chan et al. (2016) play a motivating factor for encouraging users to share resources and self-disclose personal challenges or struggles online. Despite not seeing these users in person and likely never speaking to them before, an overarching social media campaign and an additional perceived layer of privacy may in turn entice users to share supportive messages and tools with others in a safe way. With social media campaigns lending to easy and effortless participation (Jackson, 2018), users may be inclined to pass along a link or media to someone in need of help online, versus meeting up in person to talk about one's issues. The nature of Bell's CSR is thus effective in a) raising awareness; b) providing users with the illusion that they are receiving meaningful support from others; and c) encouraging users to self-disclose, though McClellan et al.'s (2017) work demonstrates that attention and interest in recurring events such as Bell Let's Talk fades quickly and therefore is not sustainable.

There was a significant drop in the volume of tweets that were categorized as 'social awareness' on the day of Bell Let's Talk (9\% where $n=21)$. Surprisingly, however, 39\% (n=26) of tweets were categorized under 'social awareness' one week before the initiative (figure 4). As mentioned earlier, many socially aware tweets tend to be advertisements. It is possible that more individual users' stories took precedence on Bell Let's Talk day as opposed to corporate advertisements promoting a brand or service. 

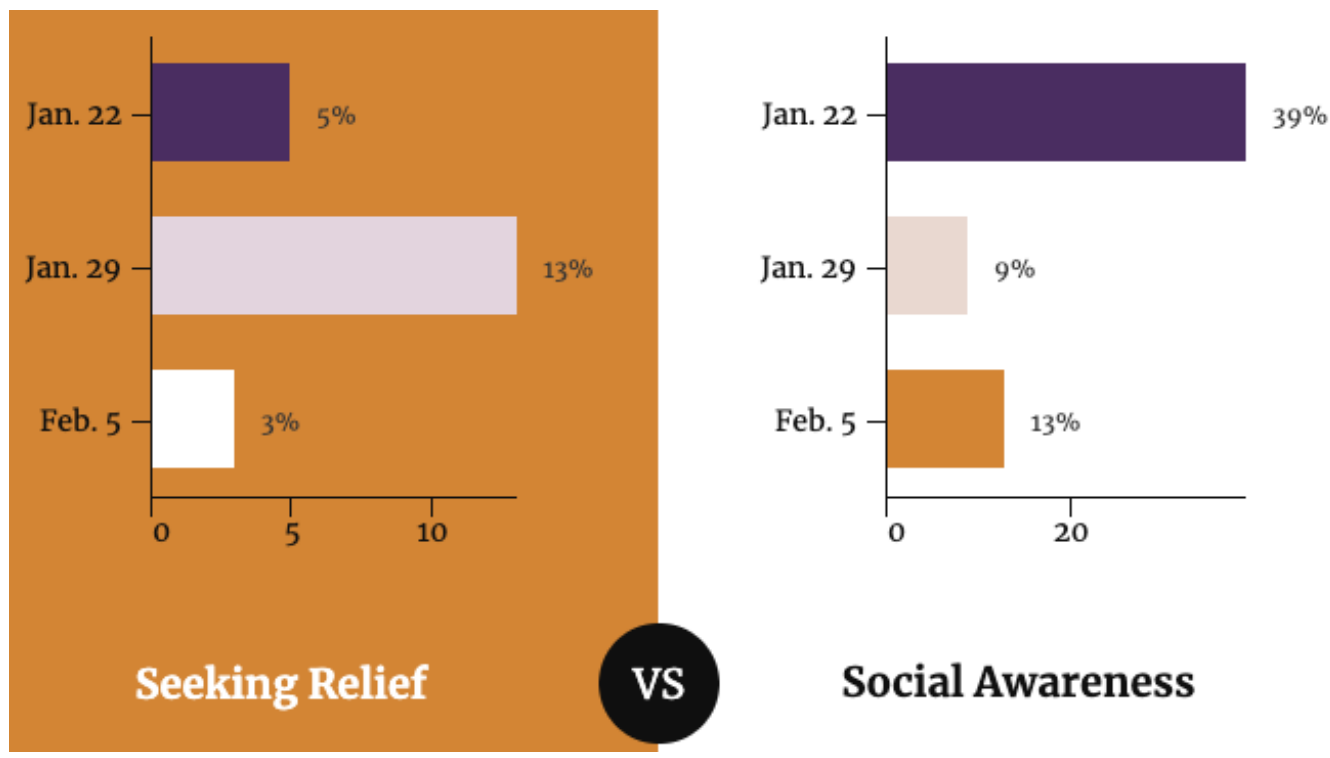

Figure 4: self-disclosure versus social awareness tweet breakdown per day

While it may be true that users self-disclose the most on Bell Let's Talk day, how many users recycle and re-share existing tweets? On the day of the initiative, only 57\% $(n=136)$ of tweets were net new ones. Forty-three (43) percent of tweets were retweets $(n=101)$. Tweet type segmentation statistics were not available for the week before and after.1 For users, retweets will still count towards the five-cent donation on Bell's part. In this case, users can feel as if they have adequately contributed to a worthy cause without exerting much effort in drafting a tweet from scratch. On a similar note, a small portion of tweets on the day of Bell Let's Talk were tweets containing only hashtags (4\% where $n=9)$. As Halupka (2014) mentions, clicktivism—as seen in the case of hashtag-only tweets and retweets especially-are low-cost, lazy forms of activism. They provide users with the illusion that they have significantly contributed to a worthwhile cause. Thus, an increase in these specific forms of tweets were prevalent on the day

1 Netlytic included all retweets, however a manual search on Twitter for tweets with \#depression returned only net new tweets. Therefore, while the retweet to net new tweet ratio was insightful on the day of Bell Let's Talk, these statistics were not available to report on one week before and after. 
of the campaign since regardless, users felt they had indirectly helped to contribute to funds for mental health programs.

Likewise, many may have tweeted resources or self-disclosing messages that could have been shared at any point in time. It is possible that most users were not tweeting for the purpose of support or opening up about one's history with depression, but simply using the hashtag \#BellLetsTalk to add to the donations towards mental health initiatives. In this case, although ephemeral due to the nature of social media, the hashtag \#BellLetsTalk creates a public forum where information is aggregated, and in turn, increases social awareness for otherwise overlooked conversation topics. It is also possible that users were completely oblivious to the fact that the campaign was running and as a result, they may have voiced opinions that could have been shared at any point in time.

To summarize, H2 was supported by the findings. Having said that, while users selfdisclosed the most on the day of Bell Let's Talk, it was not a significant enough increase from one week before to support the fact that Bell may have had a role in encouraging more personal discourse. However, it appeared that users may have taken the opportunity on the day of the initiative to seek coping mechanisms from their networks. A significant spike from one week prior demonstrates that it could be possible that Bell played a role in helping destigmatize depression and mental health more broadly, encouraging people to speak up should they need help.

\subsection{Hypothesis \#3}

Hypothesis \#3 (H3) stated that 'Bell Let's Talk day will see the highest volume of inspirational imagery attached to tweets.' In this study, inspirational imagery was classified as a) image files that were attached to a tweet; or b) a link to an Instagram post that included 
inspirational prose, quotes or affirmations (figure 5). This finding lends to the supportiveness branch that this study explores. Users may not always have an opinion to share of their own, but imagery can be just as effective as telling a story about hope or support.

\#colorado \#\#depression \#anxietysupport \#mentalillness

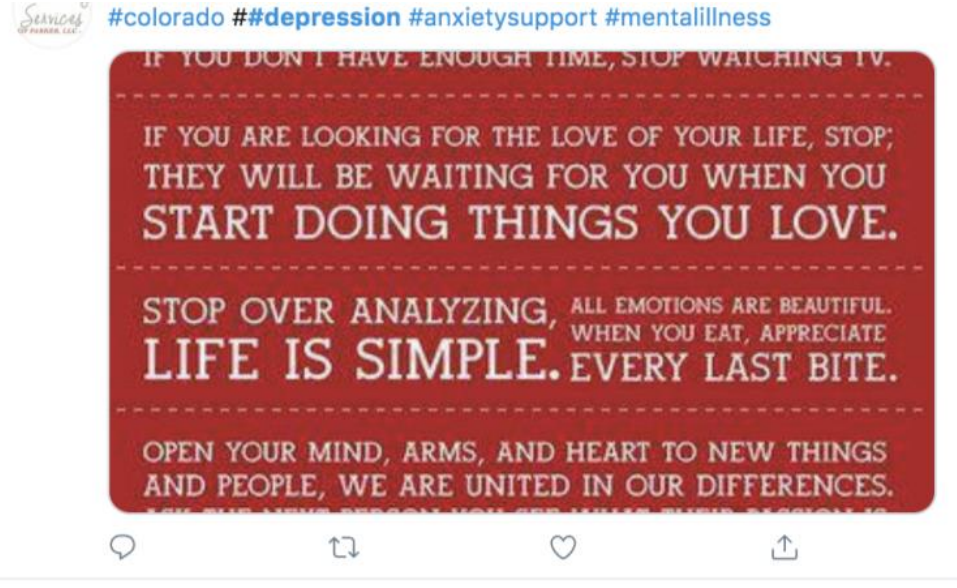

Figure 5: example of a tweet with inspirational imagery

While H1 was not supported since users did not tend to share more supportive discourse in their tweets, $\mathrm{H} 3$ was supported by the following findings. On the day of the campaign, $9 \%$ $(n=14)$ of tweets included some form of inspirational imagery. This figure is contrasted with 5\% $(n=3)$ one week before and $7 \%(n=6)$ one week after. While much of this study examines textual qualities of tweets, it was equally important to look at the mediated elements such as imagery in this case. As Blair and Abdullah (2018) point out, imagery has the power to yield interaction mirroring, whereby users may comment positive messages on optimistic posts. To assist in building a supportive community and ending the stigma around mental health, which is one of the campaign's key pillars, inspirational imagery can help facilitate supportive, meaningful discourse between users in the comment section.

Inspirational imagery can be particularly powerful when coming from an individual user as opposed to a brand or corporation. Images shared by individual users automatically feel more humanizing and may feel less promotional or sales driven. When corporations share imagery, it 
often involves a call to action to buy their product or partake in their service, demonstrating another profit-making method. When individual users share imagery and media, it is generally not for profit and instead to raise awareness or support others in creative ways.

The audience analysis in relation to the findings on the type and quantity of inspirational imagery perhaps suggests that individual users are more likely than any other group to make use of Twitter's platform affordances to share messages of hope and support. Corporations were more likely to link to their website to invite viewers to learn more about their product, but individual users were generally the group who shared supportive statements via text posts or comics.

\subsection{Hypothesis \#4}

Hypothesis \#4 (H4) stated 'Bell Let's Talk day will see the highest number of advertisements.' In this study, advertisements refer to openly sponsored and non-personal, branded communication in order to promote a service or product. Figure 6 illustrates an example of how advertising is seen via a corporation. There is often a link for users to click through to the corporation's website and there is often mention of what the corporation does and how to contact them.

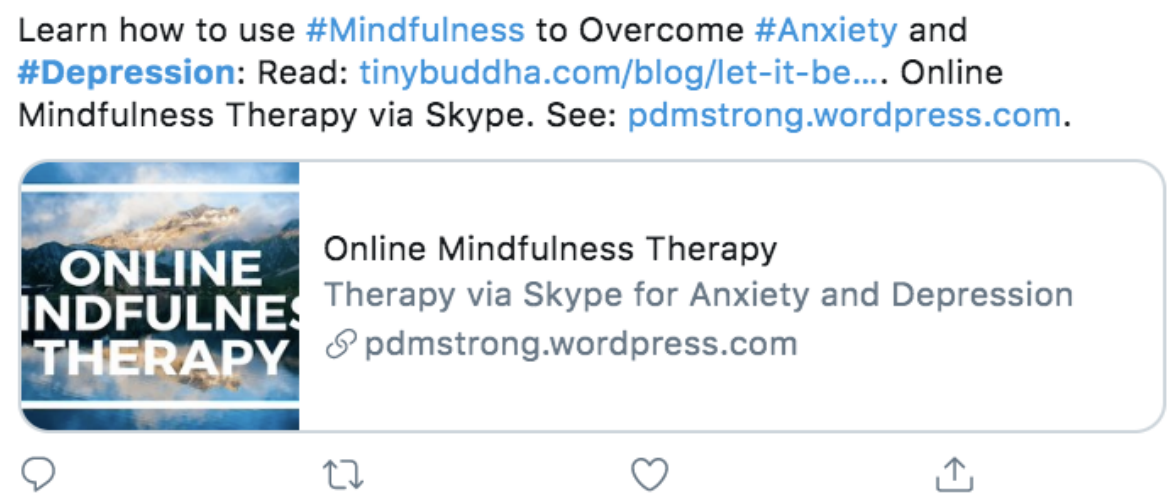

Figure 6: an example of how corporations use Twitter to advertise their services 
H4 was not supported after reviewing the findings. Contrary to expectation due to the spike in traffic around the hashtag \#depression on the day of Bell Let's Talk, this particular day had the lowest volume of advertisements recorded. In total, $15 \%(n=35)$ of users shared advertisements on the day of Bell Let's Talk. It is expected that the majority of advertisements would come from corporations promoting their products, whereas individual users would share personal thoughts and emotions. Corporations tweeted the least on Bell Let's Talk day (6\% where $n=15)$. As such, this played a role in the volume of advertisements recorded.

This volume of advertisements in tweets is compared to $26 \%(\mathrm{n}=17)$ and $21 \%(\mathrm{n}=18)$ one week before and one week after, respectively (figure 7). Advertisements do not just have to take shape in text: they can also be seen via hashtags. Across all three days, there was sustained mention of transcranial magnetic stimulation (TMS) by clinics and health care providers who offered this service. A common hashtag was \#TMS for these types of posts. Again, contrary to prior expectations, the tag \#TMS was used the least on Bell Let's Talk day, with $1 \%(\mathrm{n}=4)$ tweets using this tag. This figure is contrasted with $10 \%(n=2)$ of tweets that used the tag one week before and $2 \%(n=2)$ one week after. The significant drop in tweets with the hashtag \#TMS may be attributed to advertisers' and clinics' tweets getting lost in the sea of tweets from individual users who self-disclosed on the day of the event.

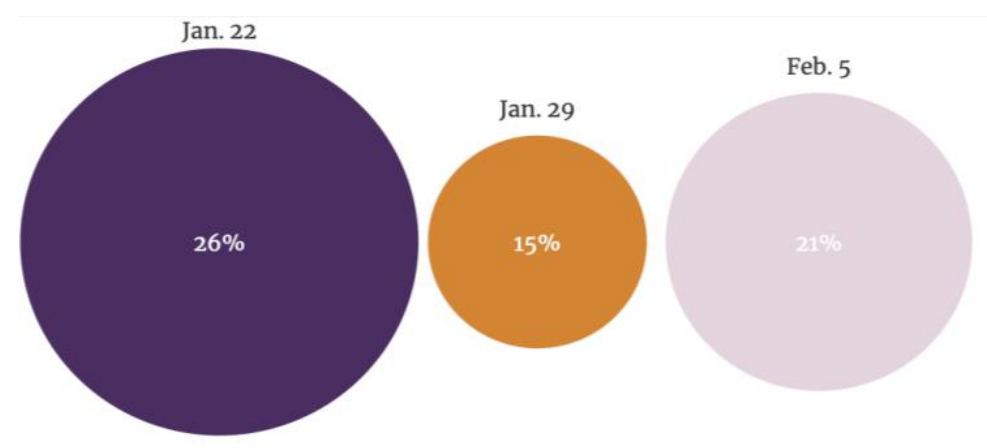

Figure 7: visual representation of promotional tweets per each day 


\section{STUDY IMPLICATIONS}

The study findings position Bell Let's Talk as an ephemeral, unsustainable social media campaign for discussing matters related to depression. While there is significant momentum around the campaign period in mid-January, Bell needs to ensure the same marketing tactics and amplification of stories around mental wellbeing are considered for later in the year as well. Doing so will better position Bell as a key player in destigmatizing depression. Mental health and depression should not be characterized as a once-per-year, sensationalized event. For instance, the findings on 'seeking relief' self-disclosure themes shows how the fewest number of tweets fell in this category one week after Bell Let's Talk, perhaps suggesting that users do not feel as comfortable sharing information and asking for help when most of the country is not participating anymore. A more frequent discussion around mental health at two or three additional points in the year would prove that Bell is not just in it for themselves but would show that they are truly invested in users' mental health and wellbeing.

Further, certain users may be more inclined to reach out for help in their communities after taking part in social media activism. Bell's 'Results and Impact' page states how much was donated to mental health initiatives, but there is no mention of how their campaign influenced users to seek help beyond the digital space. Some figures on how often, and how many, users seek professional help would help position Bell as a more sustainable, ethical campaign. 


\section{LIMITATIONS AND FUTURE RESEARCH DIRECTION}

The main limitations of this study include small sample size, inconsistent tweet format between retweets and net new tweets and technical limitations associated with Netlytic.

A small sample size can present validity issues with the dataset. Sixty-six (66) tweets were collected one week before, 237 on the day of and 87 one week later, which is presumably

only a sliver of tweets that were shared across the campaign period. Too small a sample size can increase the margin of error for this study. Contrastingly, a larger sample would provide more information about types of support and self-disclosure that users engage in, thereby reducing uncertainty around how valid or transferable the data is.

Further, there were some discrepancies with tweet format. Being that Netlytic was used on the day of Bell Let's Talk, and manual screenshots were only taken one week before and after, the tweet format differs. Netlytic included all retweets, but unknowingly, a manual scan of the hashtag \#depression on Twitter only showed net new tweets. On Bell Let's Talk day, I navigated to twitter.com and searched for the hashtag \#depression under the 'latest tweets' category, which did not show retweets. Future researchers may want to support their analysis through a third-party app like Netlytic to maximize analytic rigor and to collect a range of tweet types without any limitations. While the retweet to net new tweet ratio was insightful on the day of Bell Let's Talk, these statistics were not available to report on one week before and after.

The scope of this research can be strengthened for future iterations. Similar to McClellan et al.'s (2017) research on audience attention and discourse around expected, recurring events such as Bell Let's Talk and unexpected events, it may be useful to examine Bell's campaign efforts outside of their usual campaign period in mid-January. For instance, at the time of writing this MRP, the COVID-19 pandemic has brought with it an unprecedented number of depression 
and mental health discussions. A future study may benefit from: a) examining Bell's marketing efforts outside of their usual campaign frame; and b) understanding how discourse around mental health has shifted on social media with unexpected, global ramifications. Alternatively, a more longitudinal study spread across months and not weeks can provide more insight as to how mental health discussions shift via social media. Incorporating more checkpoints can also expose patterns in tweet content and frequency.

Moreover, the textual analysis conducted in much of this study may extend beyond the copy included in each tweet. Interaction mirroring, coined by Blair and Abdullah (2018), may be seen in each tweet's comment section. This would also lend to a better understanding of how users can support one another and form networks among themselves that transcend corporate goals. Supportiveness in this context also takes various shapes: whether that be through text or visuals. The latter is just as effective at communicating supportive messages as the former. Future research may want to center on a visual analysis of depression-related imagery or messages via Instagram or another visual-heavy platform. 


\section{CONCLUSION}

This Major Research Paper aimed to better understand how Twitter users support one another and self-disclose personal histories, stories or trajectories around depression. The research question that drove this study was 'how does the 2020 Bell Let's Talk campaign mobilize self-disclosure and social support among Twitter users?’ 390 tweets containing the hashtag \#depression across three time points were analyzed to understand how clicktivism manifests itself in mental health discourse. The findings suggest that users were not significantly more supportive of one another on the day of the event, though they appeared to self-disclose more on this date. There was also an emphasis on instrumental support on the day of Bell Let's Talk, where users offered ways get help from and to one another.

Continuing in the supportiveness branch, users evidently made use of Twitter's affordances, being the ability to share inspirational imagery or embed a link to an Instagram text post with affirmative statements. While not everyone always had something to share of their own, inspirational text posts also provided supportive, and sometimes self-disclosing, sentiment. It was also anticipated that the greatest number of advertisements would be seen on Bell Let's Talk day, which was not supported. This day had the least number of advertisements, perhaps suggesting that services or brands preferred to let individual users' stories shine.

Ultimately, the sample size of this study is too small to prove causation. Of interest though was the fact that Twitter users tend to self-disclose most on Bell Let's Talk day and from the supportiveness side, most share ways to cope and get help on this particular day. A further analysis into how Bell's campaign operates outside of the one key day will be required to understand how Bell is impacting citizens who struggle with mental health. While Bell appears to be doing a sufficient job at encouraging personal stories to be told and inviting users to share 
resources on how to alleviate their depression, this should not be taken at face value. The fact that Bell heavily profits off the campaign by incorporating their name into the hashtag, while hypocritically overlooking their mantras in unjust employee treatment shows that there is much work to be done. It is okay for Bell to talk, but they had better make time to listen as well. 


\section{APPENDIX 1}

\section{Codebook}

1) WHO (the account is a...)

1. Corporation

2. Service

3. Ordinary, individual user

4. Individual healthcare provider

5. Other

2) WHAT: Tweet types

1. Support: affirmative and encouraging tweets from one to many, or with individual users describing their personal histories and seeking help from others.

- Type of support (from Andalibi et al. (2017), p. 9) - REFER TO APPENDIX 2

2. Self-disclosure: user provides insight into their mental health history and personal trajectory in either negative or positive ways.

○ Type of self-disclosure (from Lachmar et al. (2017), p. 4) - REFER TO APPENDIX 3

3. Both supportive and self-disclosure

4. Advertisement: involves openly sponsored and non-personal, branded communication to promote a service or product.

5. Other: these tweets may involve anti-depression sentiment, or unrelated tweets with the hashtag depression used for traffic purposes.

6. Hashtag only: tweets that have no body copy except for hashtags.

3) Co-occurrence of other tags (e.g. \#depression along with \#SickNotWeak)

4) External sources or media (photo/video)

1. Link to supporting article or media for more information

2. Inspirational imagery (text post): includes a .PNG file containing inspirational prose, quotes or affirmations

3. No external article or media — whether embedded in tweet or external-is included

\section{5) Net New or Retweet}

1. Net new tweet: user drafted and shared a tweet of their own

2. Retweet: user re-shared an existing tweet on their timeline

\#colorado \#\#depression \#anxietysupport \#mentalillness

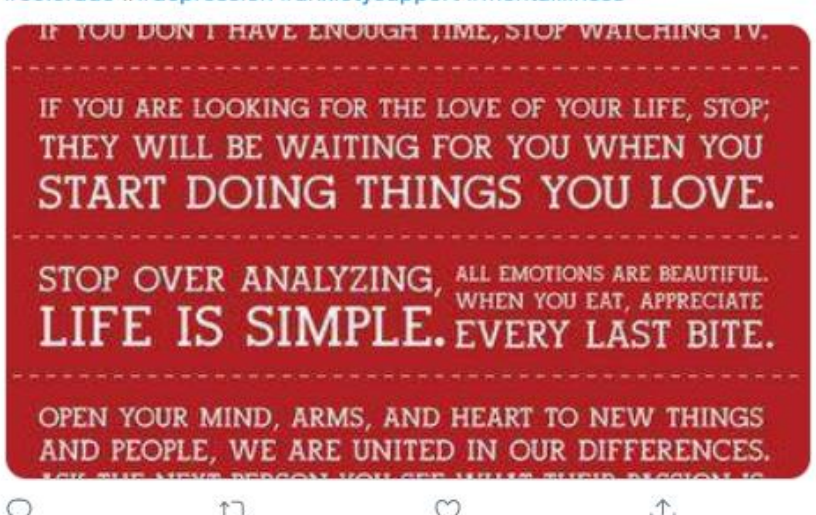

Model tweet

- Ordinary, individual user

- Emotional support

- Positive self-reflection

- Inspirational imagery 


\section{APPENDIX 2}

Type of support (per Andalibi et al. (2017)

'Support,' as defined by Andalibi et al.: building a sense of community through sharing experiences and emotions with others.

1. Informational support: advice/guidance

2. Instrumental support: ways of coping/getting help

3. Emotional support: communicate love/caring

4. Network support: belonging to a group with similar concerns

5. Esteem support: communicate respect/confidence in abilities

6. Tweet is not supportive in any way

7. Social awareness or anti-stigma 


\section{APPENDIX 3}

Type of self-disclosure (per Lachmar et al. (2017)

'Self-disclosure,' as defined by Lachmar et al.: expressing personal sentiments and insight into experiences with mental health. This area also looks at how users talk about depressive symptoms on Twitter.

Items 1-7 are provided by Lachmar et al. Items 8-11 were added in order for this study to be more comprehensive. A preliminary analysis of all tweets shows that some users have a positive self-view while some users discuss individual symptoms more frequently than others.

\section{Dysfunctional thoughts}

a. Description: thoughts about self that are negative, hopelessness, feeling unlovable

2. Lifestyle challenges

a. Description: difficulty with eating, sleeping, motivation, daily tasks

3. Social struggles

a. Description: struggles with social relationships, grapples with feelings of isolation and loneliness

4. Hiding behind a mask

a. Description: pretends to be okay in front of others; hides one's true feelings

5. Apathy and sadness

a. Description: expresses feelings of sadness and emptiness

6. Suicidal thoughts and behaviors

a. Description: descriptions of self-harm or thought of death

7. User seeks relief

a. Description: self-care and coping mechanisms. Also discusses ways to alleviate depression

8. Positive self-reflection

a. Description: user's self-view is positive and affirmative

9. Personal ailments or disorders

a. Description: details personal health challenges including disorders or ailments

10. Tweet does not include any self-disclosing information or 'other'

11. Social awareness or anti-stigma

a. Information around mental health or statistics; does not disclose any personal information. Often advertisements may fall into this category. 


\section{REFERENCES}

Andalibi, N., Ozturk, P., \& Forte, A. (2017, February). Sensitive self-disclosures, responses, and social support on Instagram: the case of \#depression. In Proceedings of the 2017 ACM conference on computer supported cooperative work and social computing (pp. 14851500). ACM. doi:10.1145/2998181.2998243

Bell Canada (n.d.). Our Initiatives. Retrieved May 16, 2020 from https://letstalk.bell.ca/en/ourinitiatives/

Bell Canada (n.d.). Results and Impact. Retrieved May 16, 2020 from https://letstalk.bell.ca/en/results-impact/

Bell Canada (n.d.). Ways to Help. Retrieved May 16, 2020 from https://letstalk.bell.ca/en/waysto-help

Blair, J., \& Abdullah, S. (2018, May). Supporting Constructive Mental Health Discourse in Social Media. In Proceedings of the 12th EAI International Conference on Pervasive Computing Technologies for Healthcare (pp. 299-303). ACM. doi:10.1145/3240925.3240930

Chan, J. K., Farrer, L. M., Gulliver, A., Bennett, K., \& Griffiths, K. M. (2016). University students' views on the perceived benefits and drawbacks of seeking help for mental health problems on the internet: A qualitative study. JMIR Human Factors, 3(1), e3. doi:10.2196/humanfactors. 4765

Gerbaudo, P. (2012). 'Friendly' Reunions: Social Media and the Choreography of Assembly. In Tweets and the Streets: Social Media and Contemporary Activism (pp. 18-47). Pluto Press. 
Halupka, M. (2014). Clicktivism: A systematic heuristic. Policy \& Internet, 6(2), 115-132. doi:10.1002/1944-2866.POI355

Ho, K. (2016). Let's Talk About How My Job at Bell Gave Me Mental Health Issues and No Benefits. Retrieved May 6, 2020 from https://www.canadalandshow.com/lets-talk-abouthow-my-job-bell-gave-me-mental-health-issues-and-no-benefits/

Jackson, E. (2018). How Bell Let's Talk became a triple win for BCE, consumers and the mental health cause. Retrieved April 2, 2020 from https://business.financialpost.com/telecom/how-bell-lets-talk-became-a-triple-win-forbce-consumers-and-the-mental-health-cause

Lachmar, E. M., Wittenborn, A. K., Bogen, K. W., \& McCauley, H. L. (2017). \#MyDepressionLooksLike: examining public discourse about depression on Twitter. JMIR mental health, 4(4). doi:10.2196/mental.8141

Magder, J. (2014). Bell Let's Talk Day: A good deed, or just good PR? Retrieved April 2, 2020 from https://montrealgazette.com/technology/bell-lets-talk-day-doesnt-sit-well-with-allcanadians

McClellan, C., Ali, M. M., Mutter, R., Kroutil, L., \& Landwehr, J. (2017). Using social media to monitor mental health discussions - evidence from Twitter. Journal of the American Medical Informatics Association, 24(3), 496-502. doi:10.1093/jamia/ocw133

McLean, M. (2017). Let's Talk About How Bell Fired Me After I Asked For Mental-Health Leave. Retrieved May 6, 2020 from https://www.canadalandshow.com/bell-lets-talkmental-health-fired/

Mental Illness \& Addiction in Canada. (2019). Mood Disorders Society of Canada. Retrieved April 2, 2020 from https://mdsc.ca/docs/MDSC_Quick_Facts_4th_Edition_EN.pdf 
Park, A. (2014). Most People With Depression Aren't Getting Treatment, Survey Finds.

Retrieved May 16, 2020 from https://time.com/3615353/depression-seeking-treatment/

Peters, M. (2018). How Bell Canada Capitalises on the Millennial: Affective Labour, Intersectional Identity, and Mental Health. Open Cultural Studies, 1(1), 395-405. doi.org/10.1515/culture-2017-0037

Razak, N. A. M. (2018). Advertising In Social Media As A New Approach In Mental Illness Campaign Among Youth. IDEALOGY, 3(2), 224-234.

Robinson, R. (2020). The 7 Top Social Media Sites You Need to Care About in 2020. https://spark.adobe.com/make/learn/top-social-media-sites/

Servera-Francés, D., \& Piqueras-Tomás, L. (2019). The effects of corporate social responsibility on consumer loyalty through consumer perceived value. Economic Research-Ekonomska Istraživanja, 32(1), 66-84. doi:10.1080/1331677X.2018.1547202

Statistics Canada. (2017, May 11). Accessing Mental Health Care in Canada. https://www150.statcan.gc.ca/n1/pub/11-627-m/11-627-m2017019-eng.htm Social Solutions (n.d.). Top 5 Barriers to Mental Healthcare Access. Retrieved July 7, 2020 from https://www.socialsolutions.com/blog/barriers-to-mental-healthcare-access/ 